\title{
Classification of FNIRS Using Wigner-ville Distribution and CNN
}

\author{
Shahriar Zaman \\ Dept. of Biomedical Engineering, Khulna University of Engineering \& Technology, Khulna, Bangladesh \\ Email: sznayeem@gmail.com

\section{Sheikh Md. Rabiul Islam} \\ Dept. Electronics and Communication Engineering, Khulna University of Engineering \& Technology, Khulna, \\ Bangladesh \\ Email: robi@ece.kuet.ac.bd
}

Received: 02 February 2021; Accepted: 28 March 2021; Published: 08 October 2021

\begin{abstract}
Consumers undergo an intellectual burden when working with technological programs. Mostly in situations of several activities. For instance, while communicating when driving with the navigation device. It is not necessary to divert users from their primary duties in such circumstances. In memory cycles and related workload, the pre-frontal cortex (PFC) has a significant role to play. In this study, we have used data from 10 participants to evaluate the task behaviors in PFC with usable near-infrared spectroscopy (fNIRS), which is a non-invasive imaging modality. In classification, CNN research has been state of the art. This has undermined the need to extract features manually. In order to assess the mental workload, we implemented a time-frequency approach with CNN approach. Rather than traditional CNN network we used ResNet50 pretrained network here. Application of Wigner-Ville Distribution in Functional Imaging is introduced here. The proposed CNN approach achieves a considerable average improvement relative to conventional methods. The results across differences in time window length are benchmarked. Satisfactory result obtained with twenty five second window for which the CNN yields $98 \%$ correct classification where traditional CNN achieved $89 \%$ accuracy.
\end{abstract}

Index Terms: FNIRS, Mental Workload, CNN, WVD.

\section{Introduction}

Human action is regulated by the consciousness of human beings. The brain is alert and conscious of anybody's activity, while various brain components are modified to effectively execute specific tasks. The activation of neurons regulates brain functions. When a given activity is carried out, the appropriate brain field is triggered. The activation needs energy supplied by blood oxygen. The process of regulation of Cerebral Blood Flow (CBF) that enables the necessary supply of oxygen to localized areas due to neural activation of brain, is known as Neurovascular Coupling. Such specific behaviors can be assessed in multiple ways including fMRI, EEG, fNIRS, and so forth [1]-[4].

One of the main factors for increasing the performance of a job is stress management. If depression can be assessed, a person's quality of life and safety may be increased [5], [6]. Many methods require facial expressions, and others include testing to measure emotional tension. These can be achieved in multiple forms. In certain cases, different physiological parameters such as breath rate, cardiac rhythm, and brain wave analysis are calculated [7], [8]. FNIRS's unique features such as inexpensive and compact printing have recently attracted significant interest in the present days.

In fNIRS, a neural function is measured by oxygen consumption in the neural and by neurovascular binding of cerebral fluid. Throughout the near-infrared range $(700-900 \mathrm{~nm})$, the absorption of hemoglobin molecules is prominent. Chromophores include hemoglobin ( $\mathrm{HbR})$, deoxyhemoglobin $(\mathrm{HbR})$, water $\left(\mathrm{H}_{2} \mathrm{O}\right)$ and cytochromes are the main light absorbing moles in the brain tissue [6], [9], [10]. The HbO and HbR concentrations shift as a consequence of brain stimulation. Using Near-infrared light (NIR), oxygenated $(\Delta \mathrm{cHbO})$ and deoxygenated hemoglobin $(\Delta \mathrm{cHbR})$ concentration are calculated. The mental cognitive states are measured using fNIRS device that directs a NIR light on Prefrontal Cortex(PFC) area and measures change of concentration of oxygenated hemoglobin $\Delta \mathrm{cHbO}(\mathrm{t})$ and concentration change of deoxy hemoglobin $\triangle \mathrm{cHbR}(\mathrm{t})$ in brain tissues using Modified Beer-Lambert law (MBLL) [6]. The operator's contact with machines and other tools is impaired by mental workload (MWL). MWL may impact the activity of the consumer, and often defense [5], by increased defect levels and response times, exhaustion and lack of so- called cognitive tunneling [11]. The cognitive characteristics and state of the user are also a crucial move in 
enhancing the architecture of human machine interface (HMI) with the introduction of adaptive software to accommodate the adjustments to mental workload [12]. In the past, different forms of tests were conducted using mental arithmetic, motor imaginary, motor execution, and other methods, to test the precision of classification systems.

In this paper, the classification of cognitive occurrences based on fNIRS requires a comparatively limited amount of training data to resolve a high-dimensional paternity classification problem [5]. Conventional methods of classification a involve preliminary selection of features before model testing. The suitable collection of features is quite complicated and the issue has been studied extensively. The vast number of features in contrast to the existence of minimal training data typically adds dimensionality to classification.

In literature reviews, have shown the results of $\mathrm{CNN}$ high classification of accuracy achieve in many applications such image registration, image processing, spece detection, and multi time-series processing. It has done properly feature extraction from any signal or image, specifically image. This CNN is considerable for fNIRS-based BCI because it is perfectly extracts the information of hemodynamic response from fNIRS signal. In this research, we counted some research question for design of research such as

- Does CNN outperform conventional methods in fNIRS-based BCI?

- How well does CNN work with the input data of the hemodynamic response signal?

- Is Wigner-Ville applicable for the hemodynamic signal?

- How different classifier performs in fNIRS signal?

- Does window length have any significance in classification accuracy? If so then what window length is best for ResNet50?

To address these questions, we need to find out how the model is the best fitting the curve in machine learning approaches in fNIRS-based BCI.

\section{Background Study}

Multiple studies were made before which have taken the advantage of CNN but there are no studies that use the time-frequency analysis to access mental workload by fNIRS data. So, our goal is to take the benefit of time-frequency analysis to access the validity of using this method in this field.

\section{A. Time-Frequency Response}

Time-frequency analysis in signal processing involves those techniques that simultaneously analyze a signal in time and frequency domains using different time-frequency representations. Instead of studying a 1-dimensional signal (a function, simple or complex-valued, whose domain is the real line) and some transform (another function whose domain is the real line, obtained from the original via some transform), time-frequency analysis studies a twodimensional signal - a function whose domain is the two-dimensional real plane, obtained from the signal via a timefrequency transformation [13], [14].

\section{B. Wigner-Ville Distribution}

As is well known, the Fourier Transform Analysis is a very useful method for analyzing stationary (timeindependent) signals (or waveforms). Nevertheless, the biomedical signals are usually non-stationary. These signals or waveforms cannot be fully analyzed by the Fourier process. Therefore, a complete study of non-stationary signals includes all time-frequency signal representations. In the year 1948, Ville proposed the Wigner distribution of a function or signal $\mathrm{f}(\mathrm{t})$ in the form

$$
W_{f}(t, \omega)=\int_{-\infty}^{\infty} f\left(t+\frac{\tau}{2}\right)+\bar{f}\left(t-\frac{\tau}{2}\right) e^{-j \omega t} d \tau
$$

for analysis of the time-frequency structures of nonstationary signals, where $\bar{f}(t)$ is the complex conjugate of $\mathrm{f}(\mathrm{t})$. Subsequently, this time-frequency representation of a signal $\mathrm{f}(\mathrm{t}$ ) is known as the Wigner-Ville distribution (WVD) which is one of the fundamental methods that have been developed over the years for the time-frequency signal analysis.

Apart from other linear time-frequency representations, such as the short-term Fourier transform (STFT) or the Gabor transform, the WVD play a central role in the field of bilinear or quadratic time-frequency representations. In view of its remarkable mathematical structures and properties, WVD is now well known as an efficient tool for timefrequency analysis of non-stationary signals and non-stationary random processes.

In recent years, this application has acted as a valuable method for research in many areas as diverse as quantum mechanics, physics, acoustics, electronics, biomedical engineering, signal processing and image processing [14]. The distribution Wigner-Ville incorporates terms of interference which almost always exacerbate its comprehension. To 
sharpen the distribution, it can use lowpass windows to filter the interpretation. The smooth Wigner-Ville pseudo distribution employs separate windows in time and frequency to filter out the interference [13], [15].

where,

$$
S P W V D_{x}^{g, H}(t, f)=\int_{-\infty}^{\infty} g(t) H(f) k(t) e^{-j 2 \pi f t} d t
$$

$$
k(t)=x\left(t+\frac{\tau}{2}\right) x^{*}\left(t-\frac{\tau}{2}\right)
$$

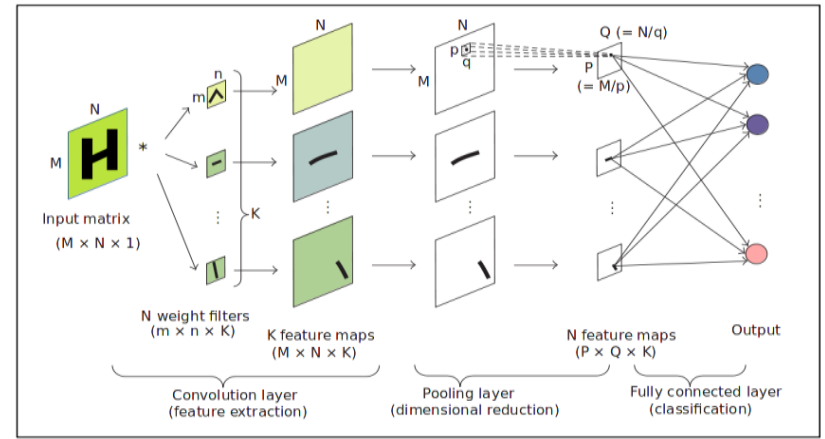

Fig. 1. Basic CNN Architecture

\section{Convolutional Neural Network(CNN)}

CNN is a type of artificial neural feed-forward network with two hidden layers, convolution, and pooling. Repetition within a wired network is used for the weights of these hidden layers. Fig 1, displays the CNN method for visual recognition. There, the image of $\mathrm{M} \times \mathrm{N}$ pixels is introduced into the convolution row. $\mathrm{K}$ number of attribute extraction filters, referred to as "kernels", is spread around the entire image using the $m \times n$ window in the convolution layer [16]. Upon translating the picture to the kernel, the convolution procedure is developed as follows:

$$
u_{i j k}=\sum_{a=0}^{m-1} \sum_{b=0}^{n-1} X_{(i+a)(j+b)} W_{a b k}
$$

where $\mathrm{x}_{\mathrm{ij}}$ is the pixel $(\mathrm{i}, \mathrm{j})$ of the input image matrix, $\mathrm{w}_{\mathrm{ijk}}$ is the weight to the pixel $(\mathrm{i}, \mathrm{j})$ of the kth kernel, and $\mathrm{u}_{\mathrm{ijk}}$ is the convoluted output with the $\mathrm{k}^{\text {th }}$ kernel at the pixel $(\mathrm{i}, \mathrm{j})$.

Then the activation function extracts the convoluted output, and this is the final output in the layer of convolution:

$$
y_{i j k}=f\left(u_{i j k}\right)
$$

In the convolution layer, eventually, $\mathrm{K}$ character maps are obtained. Ordinary artificial neural networks (ANNs) involve per-processing of the abstraction of features prior to learning; however, CNN incorporates the extraction procedure of features in its design. This is why we pick CNN as our problem classification. As stated in previous section, it is difficult to properly per-process the fNIRS data in order to extract the classification feature value. CNN is willing to do so automatically. The pooling layer then creates graphs, which are obtained from the convolution layer. In the pooling layer additional learning function information is discarded. While in previous studies various methods of pooling have been suggested, here the max pooling process has been used. In this step, $\mathrm{p} \times \mathrm{q}$ pixels (referred to as pooling size) are extracted from each feature map and the maximum value is used as a single representative output value as follows:

$$
y_{c d k}^{\prime}=\max _{(a, b) \in P_{i j k}} y_{i j k}
$$

where $P_{i j k}$ is the pooling block with pixel (i, j) at the left top of the block in the kth feature map. Then, $(M / p) \times(N / q)$ pixel image is obtained because each $\mathrm{p} \times \mathrm{q}$ pixel outputs a single output value. In other words, $\mathrm{y}_{\mathrm{cdk}}$ is the output of each pixel $(c, d)$ in the $(M / p) \times(N / q)$ image. The features of the image are retained even in the reduced size of the image. Several sets of convolution and pooling layers may be linked repeatedly. The output layer consists of a neural network that is entirely connected, similar to ordinary ANNs. The number of neurons in the output layer is equal to the number of classes in the data classification.

Each neuron in the output layer is attached to all the neurons in the previous layer (the final pooling layer) and the output is measured using (4) and the SoftMax function (5).

$$
y_{j}=\sum_{i=1}^{M / p^{N} / q} w_{i j} y_{i}^{\prime}+b_{j}
$$




$$
P_{j}=\frac{e^{u_{j}}}{\sum_{k=1}^{C} e^{u_{k}}}
$$

where $u_{j}$ is the input to the neuron $\mathrm{j}$ in the output layer, yi is an output of the pooling layer, $\mathrm{w}_{\mathrm{ij}}$ is the weight from neuron $\mathrm{i}$ in the pooling layer to neuron $\mathrm{j}$ in the output layer, $\mathrm{b}_{\mathrm{j}}$ is the bias, $\mathrm{C}$ is the number of classes in the data classification, and $\mathrm{p}_{\mathrm{j}}$ is the probability that the input data is classified to class $\mathrm{j}$.

Backpropagation is used to learn to calculate weights. That is the same method as traditional ANNs. The backing optimizes the weights of the layer in order to reduce error between the output and supervised output of the fully connected layer. The $\mathrm{CNN}$ is learned to identify the input data by repeating the forward and reverse propagation.

\section{Materials and Methods}

This study makes use of the data collected from the research work performed at Cognitive Systems Lab, Institute for Anthropomatics, Karlsruhe Institute of Technology, Karlsruhe, Germany [17].

\section{A. Dataset Description}

In this experimental set up, 10 healthy participants, of whom 4 were female participants, provided data. The dataset includes 16 fNIRS data channels. All the channels were based on the participants prefrontal cortex. Artinis Medical System has registered data of Oxymon Mark III. This method uses two $765 \mathrm{~nm}$ and $856 \mathrm{~nm}$ wave-lengths and shifts in $\mathrm{HbO}$ and $\mathrm{HbR}$ concentration at output. Four sensors and four detectors were used for prefrontal cortex calculation. The time-multiplexes of each detector are calculated in two sources situated at $3.5 \mathrm{~cm}$, resulting in a total of $8 \mathrm{HbO}$ and $\mathrm{HbR}$ channels each. The analysis of signals was at $25 \mathrm{~Hz}$. Table 1 gives the summary of the information.

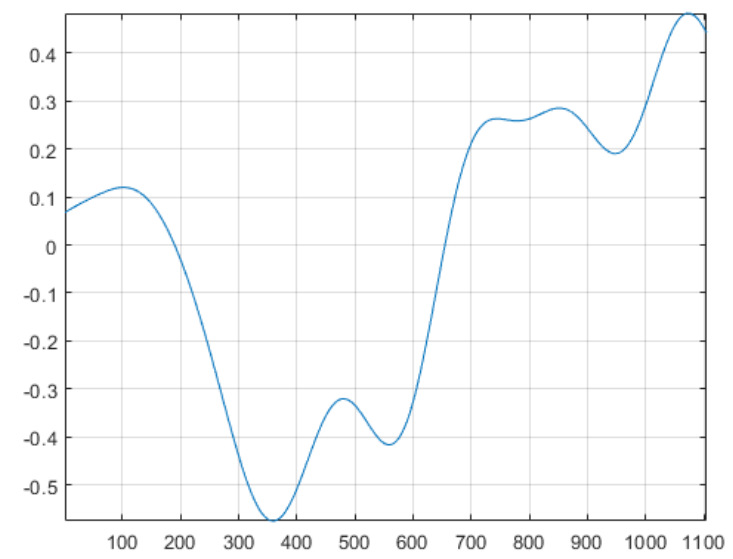

Fig. 2. Sample HbO signal (average over all channels).

Table 1. The summary of Dataset used in this study

\begin{tabular}{|c|c|}
\hline \multicolumn{2}{|c|}{ Summary of Dataset } \\
\hline No. of Subjects & 10 \\
\hline Optode Position & Prefrontal Cortex \\
\hline No. of Channels & 16 \\
\hline Wavelengths & $765 \mathrm{~nm} \mathrm{\&} \mathrm{856} \mathrm{nm}$ \\
\hline Sampling Rate & $25 \mathrm{~Hz}$ \\
\hline Performed Tasks & $\begin{array}{c}\text { n-back (1-,2- \& 3- } \\
\text { back) }\end{array}$ \\
\hline
\end{tabular}

\section{B. Experimental Setup}

This dataset includes 10 trials of each 1-, 2- and 3-back task. A trial consisted of 5s instruction, informing the subject which task (1-, 2- or 3-back) was about to start. The trial then presented a new letter every 2 s. A total of 22 letters were presented during every trial resulting in a trial length of $44 \mathrm{~s}$. Subsequently, a cross was displayed for $15 \mathrm{~s}$ during which the subjects were asked to relax ensuring that hemoglobin levels returned to baseline. After half of the trials, an additional 10 s of the resting cross were displayed to have data periods with no activity to be used as RELAX trials. A 150s break during which the subjects could drink or chat was included after 15 trials. The entire experiment had a recording time of 37 min (30 trials of 64s, 15 relax trials of $10 \mathrm{~s}$, and $150 \mathrm{~s}$ in the middle). The fNIRS data were recorded continuously during the entire session. The trials were segmented afterward based on the time sequence 
induced by the described experimental setup. Figures $3 \& 4$ have shown some experimental data representation of oxy and de-oxy hemoglobin.
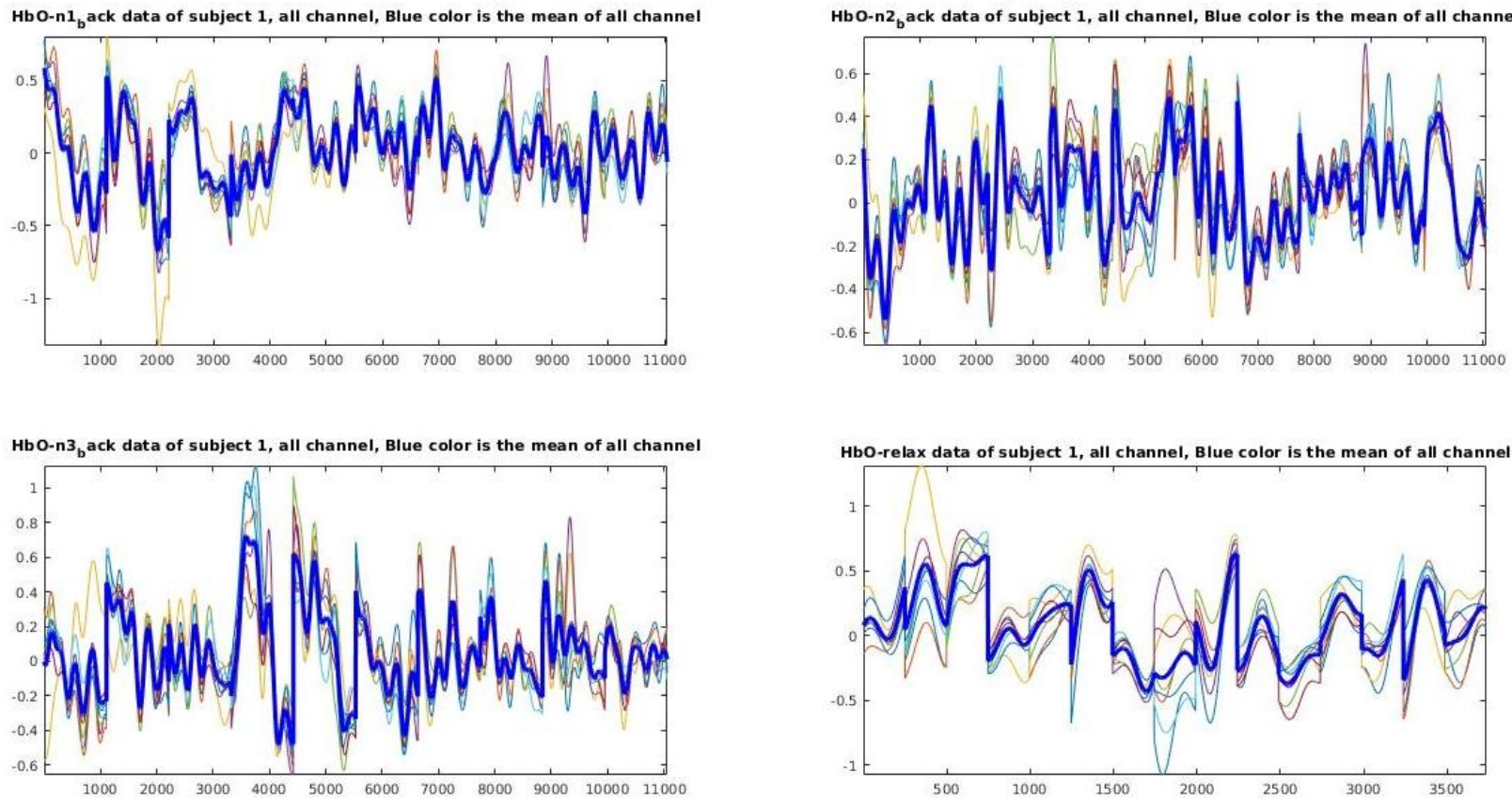

Fig. 3. Oxy Hemoglobin Data
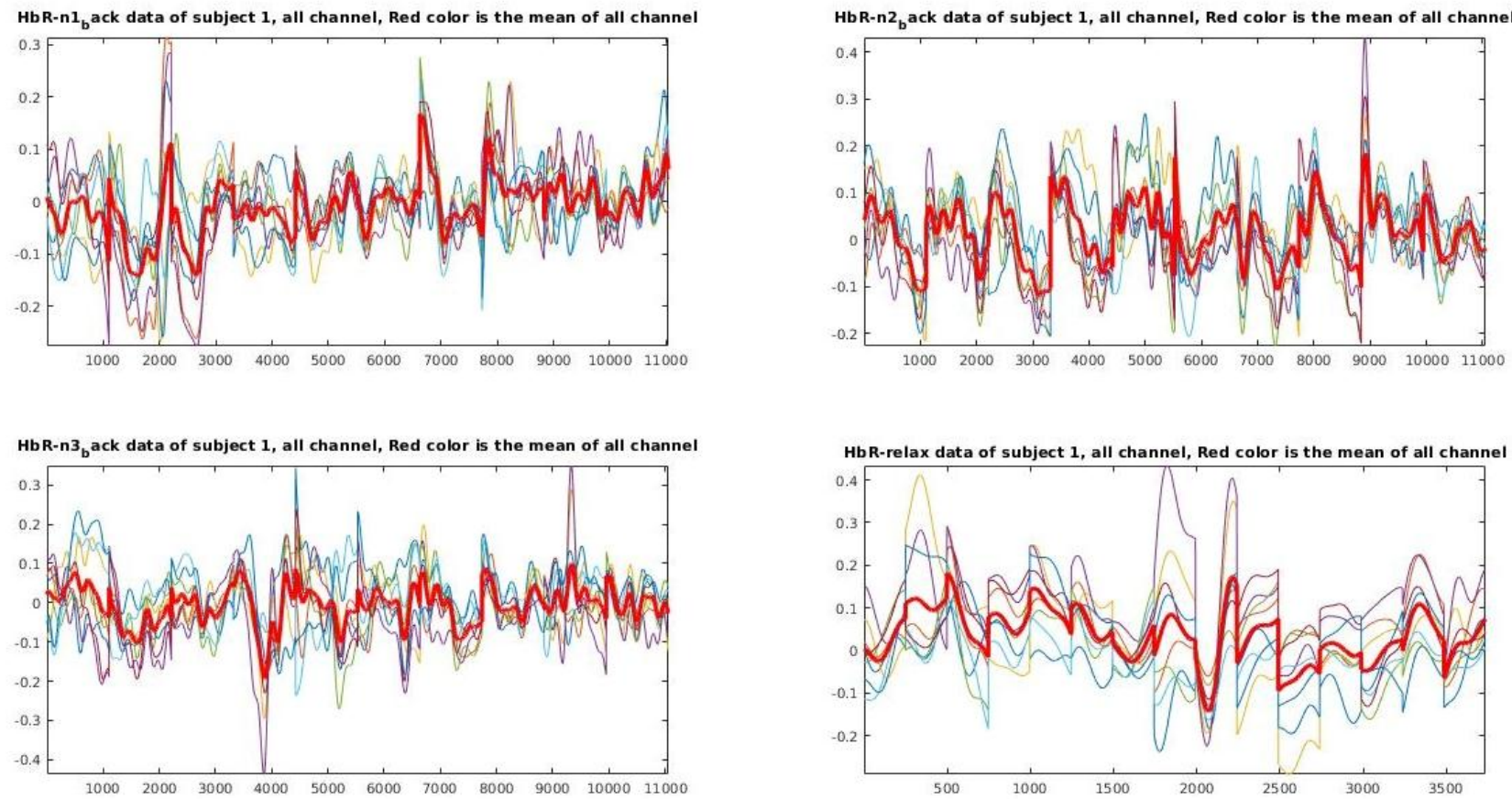

Fig. 4. Deoxy Hemoglobin Data

\section{Filtering and Baseline Correction}

The dataset contains raw fNIRS data from 10 healthy individuals. Nevertheless, fNIRS is extremely sensitive to various noises such as motion artifacts, physiological noise, cardiac rhythm and, so on. The first step is to eliminate these unnecessary signals from the $\mathrm{HbO}$ and $\mathrm{HbR}$ signal to get better results. Previous works have suggested several filter types for noise reduction. Bandpass filtering was used in this work and the passband frequency from $0.01 \mathrm{~Hz}$ to $0.13 \mathrm{~Hz}$ was chosen and the result is shown in Figure 5. 


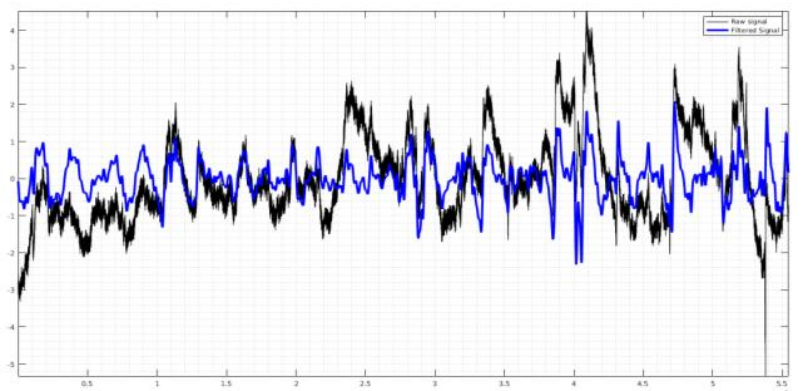

Fig. 5. Raw vs Filtered Signal

\section{Image preparation}

The signal is segmented after preprocessing according to the given data definition in Figures 6 -13 as an example. This segmented signal then produces the time-frequency response. For WVD images, different lengths of windows are considered. Study [17] indicates that window lengths of $25 \mathrm{~s}$ have the maximum degree of classification accuracy. That is the case for classifications of windows ranging from $2 \mathrm{~s}$ to $25 \mathrm{~s}$. As discussed earlier, it is often interference terms that influence WVD. This pseudo-smoothed WVD is taken into account to omit the effect of interference and, the distribution is also a threshold to zero as in Figure 14.

For classification purposes, there need images as input. The data is transformed to input using a time-frequency representation. The Wigner-Ville Distribution transforms the time series data into the 2-D image which represents the time-frequency response of the data. Different window lengths were taken into consideration. Such as $5 \mathrm{~s}$, 10s, 25s, etc. It has shown in Figure $6-13$. There is some representations where no significant output after transforming into an image. These images have not given higher accuracy in classification. So, these images are excluded from the image database.

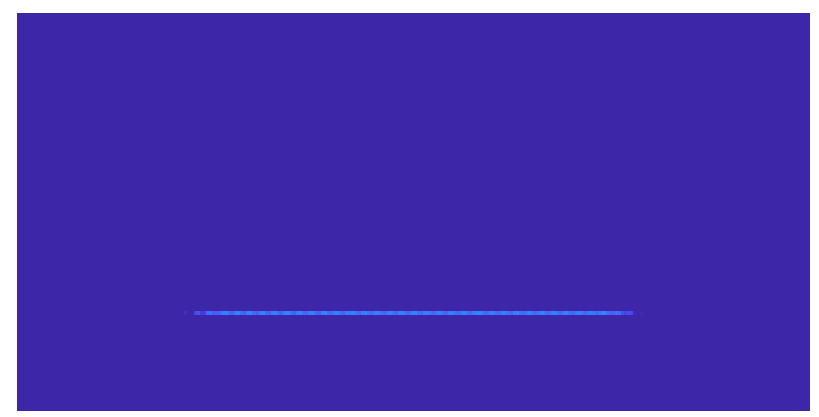

Fig. 6. WVD image of HbR data (n1-back).

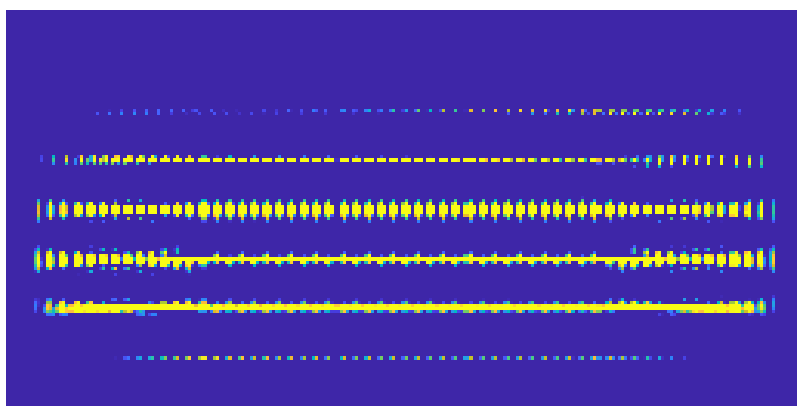

Fig. 7. WVD image of HbO data (n1-back). 


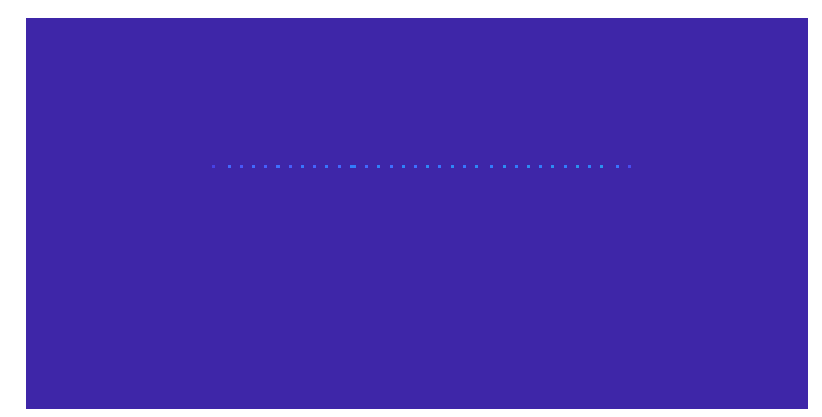

Fig. 8. WVD image of HbR data (n2-back).

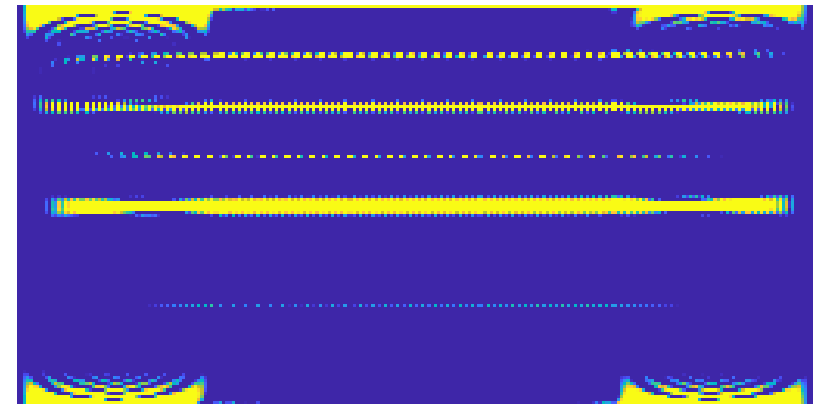

Fig. 9. WVD image of HbO data (n2-back).

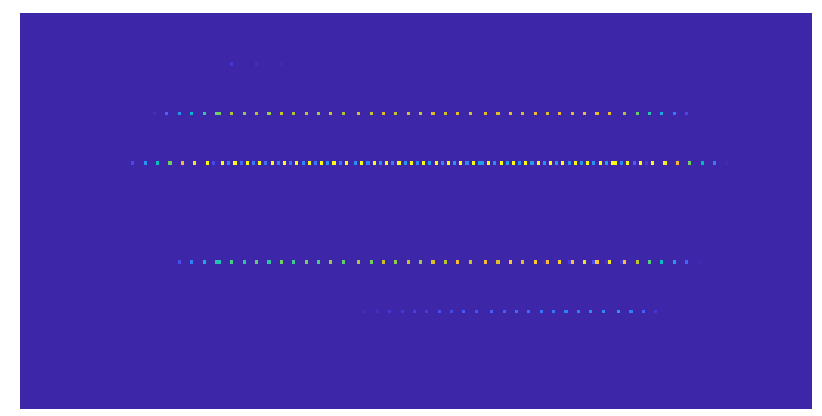

Fig. 10. WVD image of HbR data (n3-back).

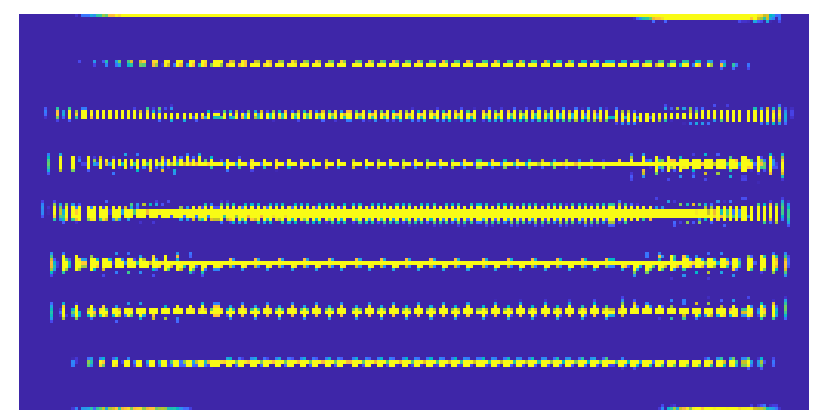

Fig.11. WVD image of HbO data (n3-back).

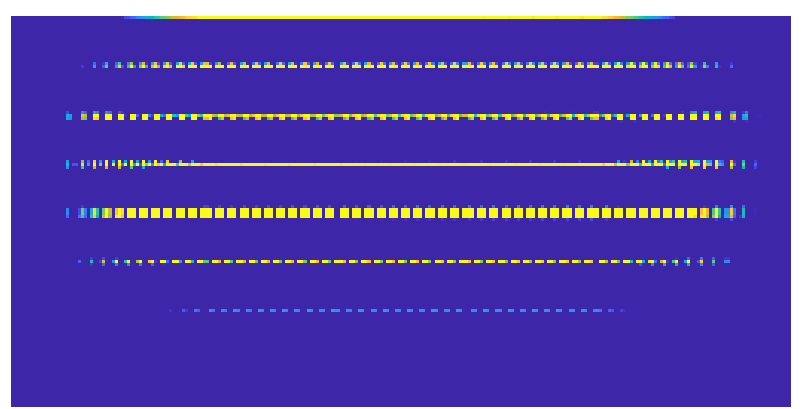

Fig.12. WVD image of HbR data (Relax). 


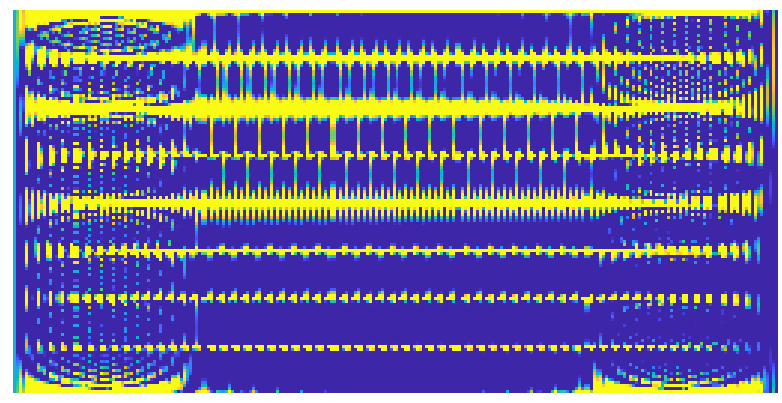

Fig. 13. WVD image of HbO data (Relax).

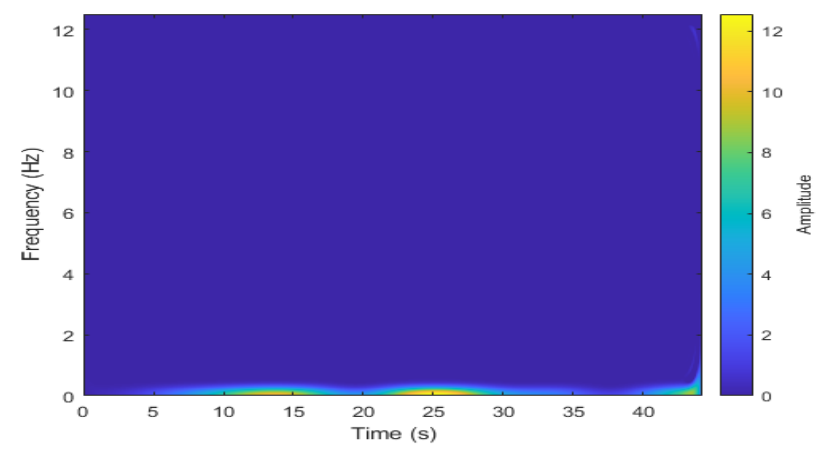

Fig. 14. Corresponding Pseudo-smoothed WVD.

\section{E. Classifier}

Convolutional Neural Network (CNN) is used to classify the mental workload. The working principle of the CNN is discussed previously in the background section. The performance between is compared afterward. This CNN model consists of four hidden layers. There are several options for the optimizer. The ADAM optimizer is chosen in these simulations.

CNN architecture is heavy in the calculation. Resnet50 has huge hidden layers inside. So, training process needs GPU support for this computational heavy architecture. At first, the training process progresses slowly. It depends on the epochs. One Epoch is when an entire dataset is passed forward and backward through the neural network only once. From the validation accuracy graph, it is obvious that till the twenty epochs there is no significant acceptable result. Again more than thirty epochs might overfit the data.

It is tested that mean pooling layer doesn't perform well. The average pooling layer shows better performance. The data is trained till fifty epochs to see how far it can go. It if found that after thirty epoch the accuracy reaches a plague state.

Rather than the epoch, the window size is more important in classification. Different window size is taken in consideration. 3-second window shows bad classification performance. Only about $73 \%$ validation accuracy. Where increasing the window length increases the accuracy level. Using 25 -second window the classifier performs the best (98\% accuracy).

There arise some cases where there is no frequency component in some images i.e. the total image is blank. These images reduce the performance of the classifier. So, for these image, an algorithm is designed that identifies which images should be written and deleted sequentially in a simulation manner. This also reduces the redundancy of images and minimizes computation time providing higher accuracy.

This is the first experimentation of classifying eight class fNIRS signas. Multi-class classification is a bit tricky. But this implementation shows that ResNet50 can be used for multi-class classification without any prior statistical analysis. This is summarized in Table 2.

Table 2.Parameters used in CNN model.

\begin{tabular}{|c|c|}
\hline \multicolumn{2}{|c|}{ Summary of CNN architecture } \\
\hline Parameters & CNN \\
\hline Optimizer & Adam \\
\hline Dropout & 0.1 \\
\hline Kernel Size & $3 \times 3$ \\
\hline Activation & ReLu \\
\hline Loss & Categorical Crossentropy \\
\hline Min Batch Size & 32 \\
\hline Epoch & 35 \\
\hline & \\
\hline
\end{tabular}




\section{Results and Discussion}

Inside the band of 0.01 to $0.13 \mathrm{~Hz}$, the data is filtered by a bandpass filter. It reduces much of the signal noise. Baseline correction was also subsequently made. Figure 14 offers an illustration of raw data and preprocessed data.

In the WVD phase, the classification only includes images that pass over the threshold. Classification of activities on specific signals has been carried out. It produces better results when considering both the HbO and HbR pictures.

For a window length of $25 \mathrm{~s}, 96.88 \%$ accuracy of the de-oxy WVD images is achieved to distinguish n3-back from relaxation. The efficiency of the $25 \mathrm{~s}$ window classification is shown in Figure 15 . The graph demonstrates that by considering multiclass classification, classification accuracy drops. Good outcomes are seen in binary classification. Better classification achieved against relaxes state. The classification accuracy comparisons of different n-back tasks with respect to relaxing state are shown in Figure 15.

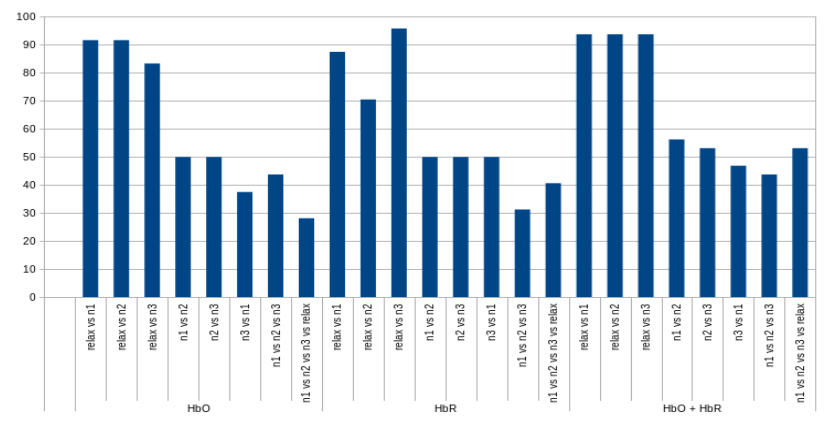

Fig. 15. Accuracy of $25 \mathrm{~s}$ window.

Though the 2 s window shows performance over $70 \%$, it is hard to tell the model can exactly classify at this performance. The accuracy of a model is not enough to predict the performance. So f1-score is also calculated. This score shows that $2 \mathrm{~s}$ window fails to classify exactly though it shows accuracy near $70 \%$. f1-score of $2 \mathrm{~s}$ window is compared in Figure 16 - 17. From this it is clear that none of the second classes are detected during test. So, the 2s window is not good for classification with WVD images.

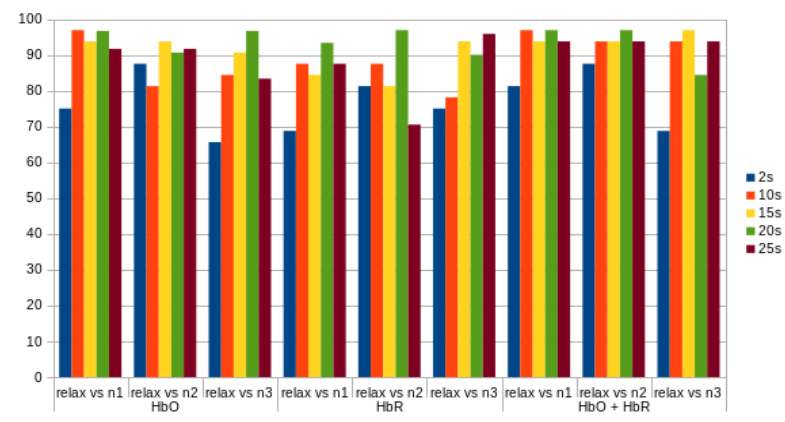

Fig. 16. Mental Workload(MWL) vs Relax performance.

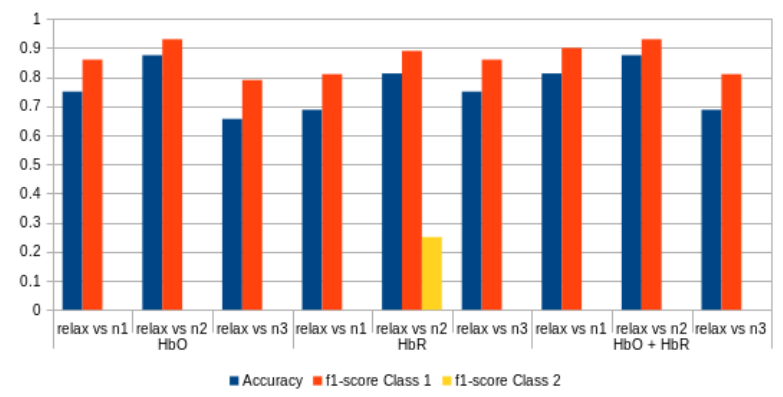

Fig. 17. Accuracy vs f1-score.

The performance improvement using CNN is showed in Figure 18. The author of this dataset used manual feature extraction method and used LDA classifier [17]. There is a significant increase in all windows. Classification accuracy increase from $70 \%$ to above $90 \%$ where all other performance also increased [17]. 


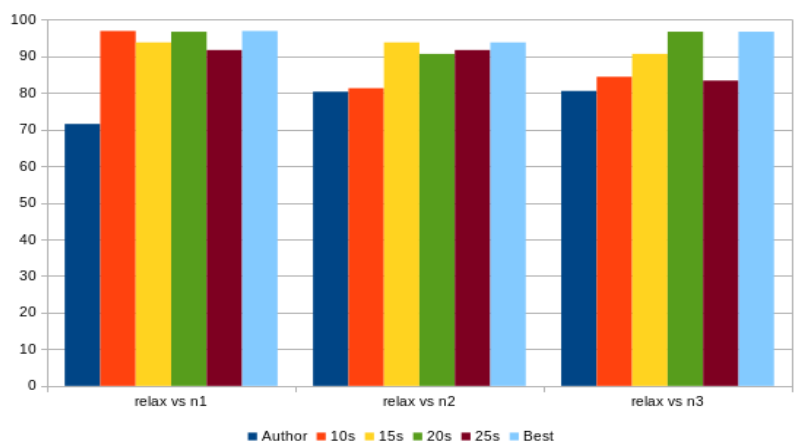

Fig. 18. Comparison with previous work.

The accuracy is mostly dependent on the window length chosen. Small window length doesn't achieve desired accuracy level. The performance over different window lengths is summarized in Table 3 and Figure 18 with the authors work and this research work.

Table 3. Result summary.

\begin{tabular}{|c|c|c|c|c|c|c|}
\hline \multirow[t]{2}{*}{ Signal } & \multirow[t]{2}{*}{ Trial } & \multicolumn{5}{|c|}{ Accuracy } \\
\hline & & $2 \mathrm{~s}$ & $10 \mathrm{~s}$ & $15 \mathrm{~s}$ & $20 \mathrm{~s}$ & $25 \mathrm{~s}$ \\
\hline \multirow{8}{*}{$\mathrm{HbO}$} & relax vs n1 & 75 & 96.875 & 93.75 & 96.67 & 91.67 \\
\hline & relax vs n2 & 87.5 & 81.25 & 93.75 & 90.62 & 91.67 \\
\hline & relax vs n3 & 65.625 & 84.375 & 90.625 & 96.67 & 83.33 \\
\hline & n1 vs n2 & 43.75 & 46.875 & 37.5 & 43.75 & 50 \\
\hline & $\mathrm{n} 2$ vs n3 & 34.375 & 49.22 & 46.875 & 56.25 & 50 \\
\hline & $\mathrm{n} 3$ vs n1 & 43.75 & 59.375 & 46.875 & 53.125 & 37.5 \\
\hline & $\mathrm{n} 1$ vs n2 vs n3 & 15.625 & 43.75 & 34.375 & 34.375 & 43.75 \\
\hline & $\mathrm{n} 1$ vs $\mathrm{n} 2$ vs $\mathrm{n} 3$ vs relax & 12.5 & 28.125 & 28.125 & 43.75 & 28.125 \\
\hline \multirow{8}{*}{$\mathrm{HbR}$} & relax vs n1 & 68.75 & 87.5 & 84.375 & 93.33 & 87.5 \\
\hline & relax vs n2 & 81.25 & 87.5 & 81.25 & 96.88 & 70.5 \\
\hline & relax vs n3 & 75 & 78.125 & 93.75 & 90 & 95.83 \\
\hline & $\mathrm{n} 1$ vs $\mathrm{n} 2$ & 50 & 53.125 & 46.875 & 50 & 50 \\
\hline & $\mathrm{n} 2$ vs $\mathrm{n} 3$ & 46.875 & 46.875 & 43.75 & 43.75 & 50 \\
\hline & $\mathrm{n} 3$ vs n1 & 43.75 & 46.875 & 46.875 & 50 & 50 \\
\hline & $\mathrm{n} 1$ vs $\mathrm{n} 2$ vs $\mathrm{n} 3$ & 34.375 & 31.25 & 21.875 & 25 & 31.25 \\
\hline & $\mathrm{n} 1$ vs $\mathrm{n} 2$ vs $\mathrm{n} 3$ vs relax & 28.125 & 43.75 & 18.75 & 31.25 & 40.625 \\
\hline \multirow{8}{*}{$\begin{array}{c}\mathrm{HbO} \\
+ \\
\mathrm{HbR}\end{array}$} & relax vs n1 & 81.25 & 96.875 & 93.75 & 96.875 & 93.75 \\
\hline & relax vs n2 & 87.5 & 93.75 & 93.75 & 96.875 & 93.75 \\
\hline & relax vs n3 & 68.75 & 93.75 & 96.875 & 84.375 & 93.75 \\
\hline & $\mathrm{n} 1$ vs n2 & 65.625 & 68.75 & 46.875 & 43.75 & 56.25 \\
\hline & $\mathrm{n} 2$ vs n 3 & 46.875 & 50 & 43.75 & 56.25 & 53.125 \\
\hline & n3 vs n1 & & 65.625 & 56.25 & 53.125 & 46.875 \\
\hline & $\mathrm{n} 1$ vs $\mathrm{n} 2$ vs $\mathrm{n} 3$ & & 34.375 & 40.625 & 25 & 43.75 \\
\hline & $\mathrm{n} 1$ vs $\mathrm{n} 2$ vs $\mathrm{n} 3$ vs relax & & 40.625 & 53.125 & 34.375 & 53.125 \\
\hline
\end{tabular}

Table 4 show the main comparisons of the different model used in [5] and this research model. It is clearly shown that this research model is very efficient as compared with average numerical accuracy value is $98 \%$. The authors [5] shown that hybrid CNN with fNIRS has shown 89\%. So , CNN with ResNet50 have shown more support for this research work. So, the performance of $2 \mathrm{~s}, 10 \mathrm{~s}, 15 \mathrm{~s}, 20 \mathrm{~s}$, and, $25 \mathrm{~s}$ also tested and the $25 \mathrm{~s}$ window gives the highest classification accuracy. It gives the highest accuracy in classification.

Table 4. Comparison between the classification accuracy of the classifier for all subjects with other model.

\begin{tabular}{|l|c|}
\hline \multicolumn{1}{|c|}{ Model } & Average Accuracy \\
\hline fNIRS with CNN [5] & $81 \%$ \\
\hline HbO with CNN [5] & $71 \%$ \\
\hline EEG with CNN [5] & $66 \%$ \\
\hline HbO+EEG with CNN [5] & $76 \%$ \\
\hline fNIRS with Hybrid SVM [5] & $82 \%$ \\
\hline fNIRS with Hybrid CNN [5] & $89 \%$ \\
\hline fNIRS with sLDA [18] & $66.08 \%$ \\
\hline fNIRS with ANN [18] & 64.02 \\
\hline fNIRS with Bi-LSTM [18] & $79.88 \%$ \\
\hline fNIRS with CNN [18] & $87.01 \%$ \\
\hline fNIRS-WVD-CNN [This research work] & $\mathbf{9 8 \%}$ \\
\hline
\end{tabular}



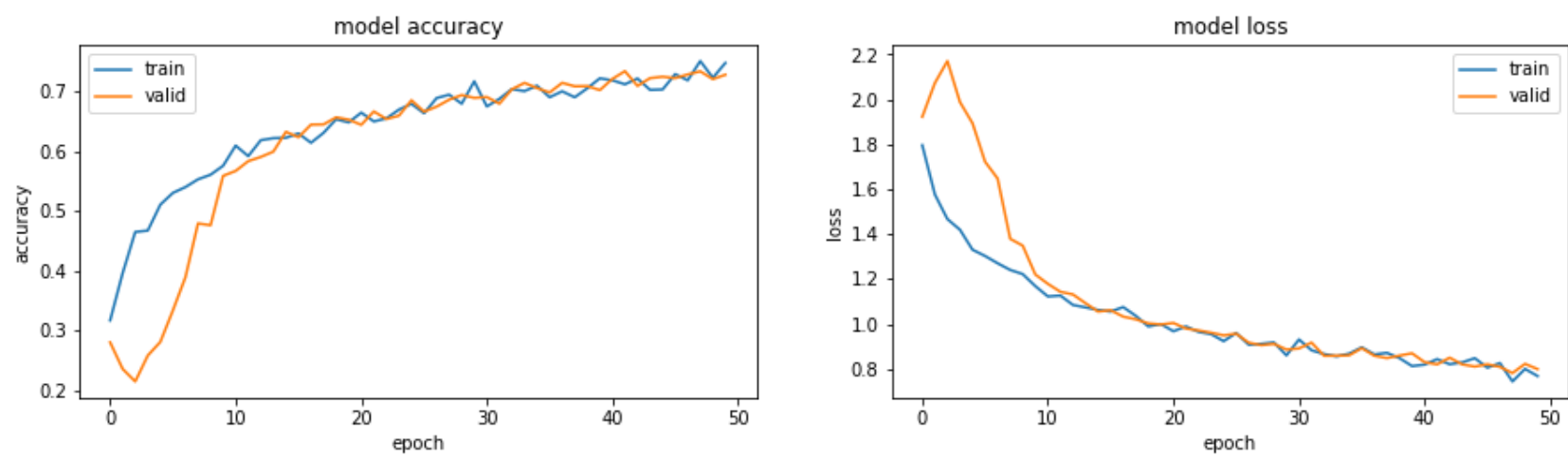

Fig.19. Test validation accuracy and loss of model for 3s window.
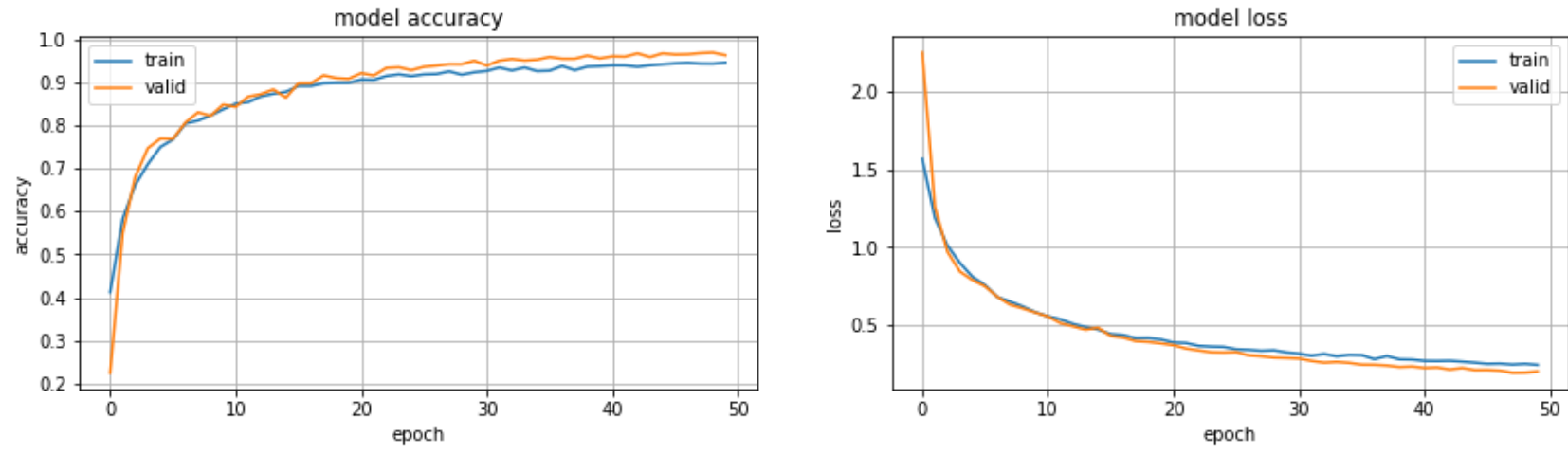

Fig. 20. Test validation accuracy and loss of model for 10s window.
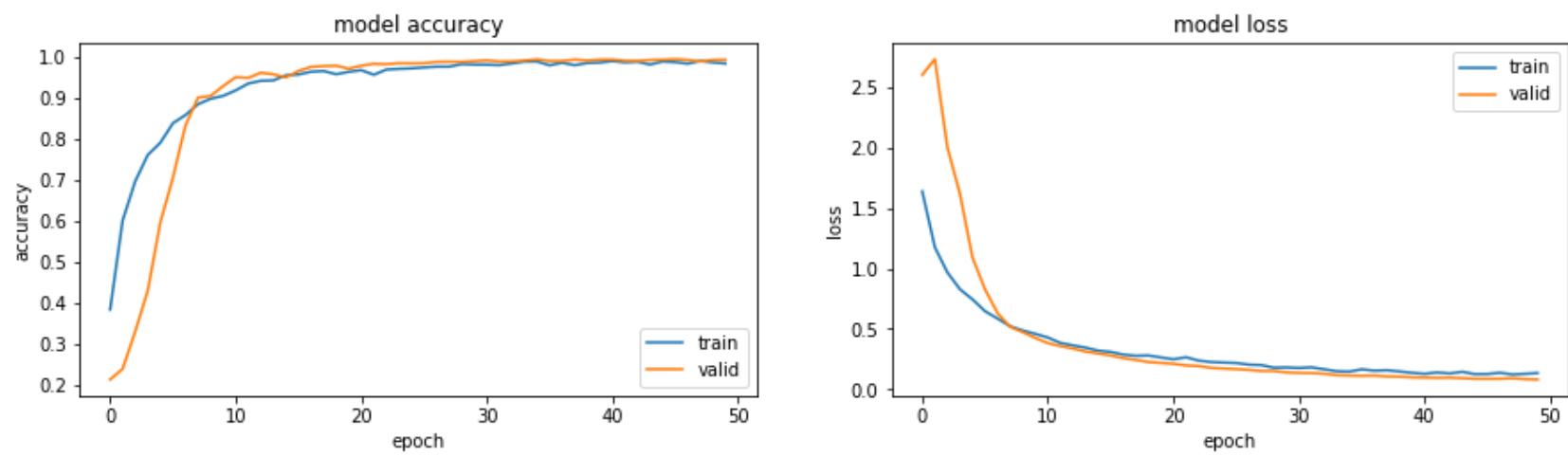

Fig. 21. Test validation accuracy and loss of model for $25 \mathrm{~s}$ window.

In model analysis, we have seen that a model can be fit depends on the different matrices. The loss function and accuracy function is accounted for in this paper. The accuracy of a model has already described above figures. Figure 19-21 (left side) shows the accuracy of learning cuve. It shows the accuracy values gradually increase based on the training and validation curve. The other function of loss in a model is calculated on training and validation sets and its interpretation are based on how well the model is doing in these two sets. Figure 19 shows the sum of errors made for ple in training or validation sets. Loss value implies how poorly or well a model behaves after each iteration of optimization. Figure 19 shows the accuracy of the model in the left side and the loss of model on the right side. After starting of epochs, the loss values is decreasing and but trained and validation decreasing with oscillates, so the model is learning at all for the $3 \mathrm{~s}$ window. On the contrary, Figures 20 and 21 have quasi-similar fashion only changed in oscillation both the validation and training curve. So, it has been shown that if we increase window size with time (10s and $25 \mathrm{~s}$ in Figure $20 \& 21)$ the training and validation curve gradually decreasing and smooth. The interpretation accuracy and loss model is showing that proposed model is the best fit as comparing to other research [5] \& [18]. The results are also shown in Table 5. 


\section{Conclusion \& Future Work}

We thus present an opportunity of fNIRS as a paradigm for passive BCI and user-state monitoring, light of the fact that more exploration is required to distinguish with greater accuracies between more degrees of workload. Clear optode mounting and solid performance enables the use of fNIRS in real-life settings such as car navigation and classroom setups. Therefore, the findings provided illustrate the viability of using fNIRS to measure workload in a single study.

\section{Acknowledgment}

The authors wish to thank Dept. of Biomedical Engineering staffs and all professor of this dept for suporting euipments and other facilities. This work was not supported in part by a grant.

\section{Conflict of interest}

The authors wish to thank Dept. of Biomedical Engineering staffs and all professor of this dept for suporting euipments and other facilities. This work was not supported in part by a grant.

\section{References}

[1] J. Shin and J. Jeong, "Multiclass classification of hemodynamic responses for performance improvement of functional nearinfrared spectroscopy-based brain-computer interface," J. Biomed. Opt., vol. 19, no. 6, p. 067009, Jun. 2014, doi: 10.1117/1.jbo.19.6.067009.

[2] N. Naseer and K. S. Hong, “fNIRS-based brain-computer interfaces: A review," Front. Hum. Neurosci., vol. 9, no. JAN, Jan. 2015, doi: 10.3389/fnhum.2015.00003.

[3] B. Blankertz et al., "The Berlin Brain-Computer Interface: EEG-Based Communication Without Subject Training," IEEE Trans. Neural Syst. Rehabil. Eng., vol. 14, no. 2, pp. 147-152, Jun. 2006, doi: 10.1109/TNSRE.2006.875557.

[4] T. Pistohl, T. Ball, A. Schulze-Bonhage, A. Aertsen, and C. Mehring, "Prediction of arm movement trajectories from ECoGrecordings in humans," J. Neurosci. Methods, vol. 167, no. 1, pp. 105-114, Jan. 2008, doi: 10.1016/j.jneumeth.2007.10.001.

[5] M. Saadati,J. Nelson, H. Ayaz, 'Convolutional Neural Network for Hybrid fNIRS-EEG Mental Workload Classification', In: Ayaz H. (eds) Advances in Neuroergonomics and Cognitive Engineering. AHFE 2019. Advances in Intelligent Systems and Computing, vol 953. Springer, Cham. https://doi.org/10.1007/978-3-030-20473-0_22

[6] U. Asgher, R. Ahmad, N. Naseer, Y. Ayaz, M. J. Khan, and M. K. Amjad, "Assessment and Classification of Mental Workload in the Prefrontal Cortex (PFC) Using Fixed-Value Modified Beer-Lambert Law,” IEEE Access, vol. 7, pp. 143250-143262, 2019, doi: 10.1109/ACCESS.2019.2944965.

[7] C. T. Gloria and M. A. Steinhardt, "Relationships Among Positive Emotions, Coping, Resilience and Mental Health," Stress Heal., vol. 32, no. 2, pp. 145-156, Apr. 2016, doi: 10.1002/smi.2589.

[8] G. Durantin, J. F. Gagnon, S. Tremblay, and F. Dehais, "Using near infrared spectroscopy and heart rate variability to detect mental overload,” Behav. Brain Res., vol. 259, pp. 16-23, Feb. 2014, doi: 10.1016/j.bbr.2013.10.042.

[9] F. Jobsis, "Noninvasive, infrared monitoring of cerebral and myocardial oxygen sufficiency and circulatory parameters," Science (80-. )., vol. 198, no. 4323, pp. 1264-1267, Dec. 1977, doi: 10.1126/science.929199.

[10] S. Cinciute, "Translating the hemodynamic response: why focused interdisciplinary integration should matter for the future of functional neuroimaging," PeerJ, vol. 7, p. e6621, Mar. 2019, doi: 10.7717/peerj.6621.

[11] J. Jarmasz, C. M. Herdman, and K. R. Johannsdottir, "Object-based attention and cognitive tunneling," J. Exp. Psychol. Appl., vol. 11, no. 1, pp. 3-12, 2005, doi: 10.1037/1076-898X.11.1.3.

[12] H. Aghajani, M. Garbey, and A. Omurtag, "Measuring mental workload with EEG+fNIRS," Front. Hum. Neurosci., vol. 11, no. July, pp. 1-20, 2017, doi: 10.3389/fnhum.2017.00359.

[13] L. Cohen, Time-Frequency Analysis. Prentice-Hall, 1995.

[14] L. Debnath and F. A. Shah, Wavelet Transforms and Their Applications. Boston, MA: Birkhäuser Boston, 2015.

[15] Mallat. Stephane, A Wavelet Tour of Signal Processing, 2nd ed.

[16] S. Hiwa, K. Hanawa, R. Tamura, K. Hachisuka, and T. Hiroyasu, "Analyzing brain functions by subject classification of functional near-infrared spectroscopy data using convolutional neural networks analysis," Comput. Intell. Neurosci., vol. 2016, 2016, doi: 10.1155/2016/1841945.

[17] C. Herff, D. Heger, O. Fortmann, J. Hennrich, F. Putze, and T. Schultz, "Mental workload during n-back task-quantified in the prefrontal cortex using fNIRS," Front. Hum. Neurosci., vol. 7, no. JAN, pp. 1-9, 2014, doi: 10.3389/fnhum.2013.00935.

[18] D. Sajila,W. M. S. Mahmud, 'A Deep Learning Based Ternary Task Classification System Using Gramian Angular Summation Field in fNIRS Neuroimaging Data',arViv:2101.05891 [cs.LG],14 Jan,2021 


\section{Authors' Profiles}

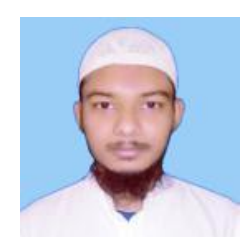

Shahriar Zaman has fulfilled his BSc degree from Khulna University of Engineering and Technology, Khulna, Bangladesh in Biomedical Engineering.

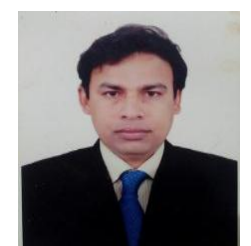

Sheikh Md. Rabiul Islam received the B.Sc. In Engineering (ECE) Khulna University, Bangladesh in December 2003, and M.Sc. in Telecommunication Engineering from the University of Trento, Italy, in October 2009 and Ph.D. from University of Canberra, Australia, in 2015. He joined as a Lecturer in the department of Electronics and Communication Engineering of Khulna University of Engineering \& Technology, Khulna, in 2004, where he is joined an Assistant Professor in the same department in the effect of 2008. Also he joined Associate Professor in 2015 and joined as Professor in 2018 in the same department. He has given four M.Sc. Engineering at Dept. of ECE and one M.Sc. Engineering (BME) at Dept of Biomedical Engineering. He is also completed four projects by funded university grant commission. Now He has published 2 book chapters, 30 Journal and 33 International conferences. His research interests include biomedical signal \& image processing, VLSI for biomedical signal and image processing, Machine learning, Embedded system. He is an IEEE member. Previous publications may be included. Try not to list more than three books or published articles. The format for listing publishers of a book within the biography is: title of book (city, state: publisher name, year) similar to a reference. Current and previous research interests end the paragraph.

How to cite this paper: Shahriar Zaman, Sheikh Md. Rabiul Islam, "Classification of FNIRS Using Wigner-ville Distribution and CNN", International Journal of Image, Graphics and Signal Processing(IJIGSP), Vol.13, No.5, pp. 1-13, 2021.DOI: $10.5815 /$ ijigsp.2021.05.01 